\title{
Half ALPACA and its sensitivity to sub-PeV gamma rays from the Galactic Center
}

\section{Yoshichika Yokoe ${ }^{a, *}$ on behalf of the ALPACA Collaboration}

(a complete list of authors can be found at the end of the proceedings)

${ }^{a}$ Institute for Cosmic Ray Research, University of Tokyo, Kashiwa, Chiba 277-8582, Japan

E-mail: yyokoe@icrr.u-tokyo.ac.jp

The Andes Large area PArticle detector for Cosmic ray physics and Astronomy (ALPACA) project is under construction near the Chacaltaya mountain at an altitude of 4,740 $\mathrm{m}$ in Bolivia. ALPACA has an $82800 \mathrm{~m}^{2}$ surface detector with 401 scintillation detectors. The construction of the half ALPACA, which is an experiment of the half density of air shower array using 200 scintillation detectors, is scheduled for 2022. The cover area of the half ALPACA is the same as ALPACA:82800 $\mathrm{m}^{2}$. In addition to this surface array, underground muon detectors cover $3600 \mathrm{~m}^{2}$ which allows us to identify muon components in the air showers. Using this array, half ALPACA will be cable of exploring sub-PeV gamma-ray sky first time in the Southern hemisphere, including the Galactic Center. The performance of the future half ALPACA observatory derived from detailed Monte Carlo simulations is presented in this contribution.

$37^{\text {th }}$ International Cosmic Ray Conference (ICRC 2021)

July 12 th - 23rd, 2021

Online - Berlin, Germany

\footnotetext{
*Presenter
} 


\section{Introduction}

Galactic cosmic rays reach energies of at least a few PeV, suggesting that our Galaxy contains $\mathrm{PeV}$ accelerating objects called PeVatron. The hadronic part of the cosmic rays which PeVatron accelerated, interacts with the interstellar gas and produces neutral pions $\left(\pi^{0}\right)$. Neutral pions decay into two photons $(\gamma \gamma)$. Those photons generated in that process are expected to have $100 \mathrm{TeV}$ (sub-PeV) energy ,because of having 10 percent of the energy of neutral pion. Besides, the photons emitted through this mechanism have a power law spectrum with the same index as the cosmic rays, protons, and nuclei. It is essential to study the sub-PeV gamma-ray diffuse emission because, they trace the overall distribution of cosmic ray protons in the Galaxy and suggest the position of PeVatron[1].

A high duty cycle and wide field of view of ALPACA(half ALPACA) is suitable for sub-PeV gamma-ray observation because of low gamma-ray flux. Alternatively, the conventional air shower array's rejection power of the enormous cosmic ray background events is not high enough. However, with the muon detector array whose structure is similar to ALPACA(half ALPACA), the TibetAS $\gamma$ experiment in the northern hemisphere can reject background cosmic ray events with 99.9 percent around $100 \mathrm{TeV}$ region. Especially, TibetAS $\gamma$ can accomplish that the few hundreds $\mathrm{TeV}$ region is background free[2].

One of our main interests is the detection of gamma rays beyond $100 \mathrm{TeV}$ from the Galactic center. In 2016, H.E.S.S observed the diffuse gamma-rays around the Galactic center at the TeV energy range[3]. Then, H.E.S.S reported hard gamma-ray emission without a break-up. This data suggests that cosmic ray accelerator exists around it, but the H.E.S.S data is up to few tens TeV. After that H.E.S.S observation, MAGIC(2020) and VERITAS(2016) observed diffuse gamma-ray emission of Galactic center up to a few ten TeV[4][5].

\section{Monte Carlo simulations and analysis}

The performance values for half ALPACA presented in the following are derived from detailed Monte Carlo(MC) simulations of the half ALPACA instrument based on the CORSIKA air shower code. The air shower generation area is inside of the circle with a $300 \mathrm{~m}$ radius. The point source moving on the orbit of the Galactic Center is adopted as a gamma-ray source. Besides, we assume to observe a gamma-ray source with a spectral shape following a power law with $E^{-2.32}$. As presented below(integral flux), the results are heavily dependent on the assumed spectral index.Background cosmic ray chemical composition assumed is Shibata model [6] with CORSIKA. The hadron interaction model is used EPOS-LHC at high energy and FLUKA at low energy.

Detector response simulations are performed with GEANT. Muon detector and AS detector constructions are presented Figure2 left and right respectively. These layouts are above(Figure1). GEANT simulates the passage of particles through matter, allowing for arbitrary geometry and materials. In air shower array instruments simulation, $94 \mathrm{MeV}$ energy deposit of scintillating 


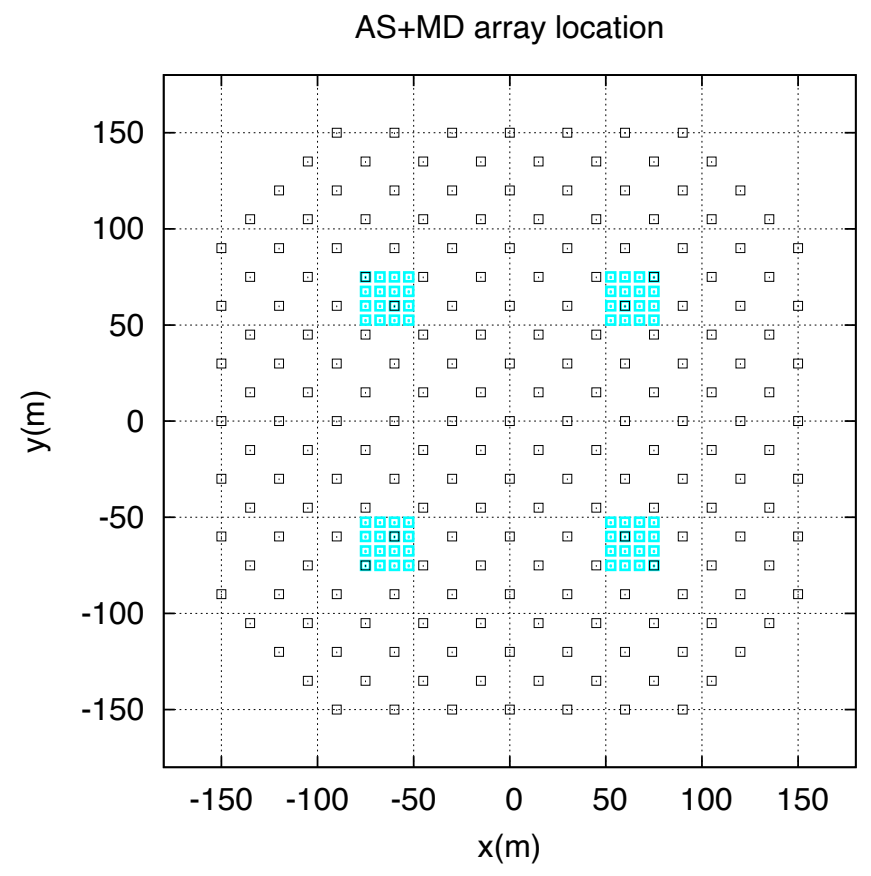

Figure 1: The half ALPACA layout. Black square shows air shower instruments and blue square shows muon detector array. Constructions of each detectors are Figure2 left and right.

detector is regarded as 1 particle. Similarly, 23 photoelectrons in the muon detector seem like 1 muon.

In this contribution, energy reconstruction is imported with the number of particles detected. (3) The timing distribution of each air shower array instruments detecting particle $\left(t_{i}\right)$ is used for estimation of incoming direction. In this method, $t_{i}$ is corrected as $t_{i, c o r}, t_{i, c o r}=t_{i}-T(R)$.Here, $T(R)$ is the function of distance $R$ from the center of air shower front cone curve. Then, we introduce the value $\left(\chi^{2}\right)$ called "residual error" (Eq. 1). Also, $l$, the incoming direction cosine, defined as minimum $\chi^{2}$.

$$
\chi^{2}=\Sigma_{i} w_{i}\left(l \cdot x_{i}-c\left(t_{0}-t_{i}\right)\right)\left(w_{i}=\frac{\rho_{i}}{\Sigma_{i} \rho_{i}}, x_{i}=\left(x_{i}, y_{i}, 0\right) c=\text { speed of light }\right)
$$

The following conditions are adopted as analysis conditions.

1 any four scintillation detectors record more than 0.8 particle density 2 the detector that records the most significant particle density is inside the inner area which is inside the air shower array, excluding the most outside instruments 3 the residual error in Equation1 is smaller than 1 meter 4 reconstructed zenith angle is smaller than $40^{\circ}$. 
5 there is the Galactic center inside the window of radius $r_{\text {win }}=\frac{5.8^{\circ}}{\sqrt{\Sigma \rho}}$. (if $r_{\text {win }}<0.5^{\circ}, r=0.5^{\circ}$ and if $r_{\text {win }}>1.5^{\circ}, r_{\text {win }}=1.5^{\circ}$ )
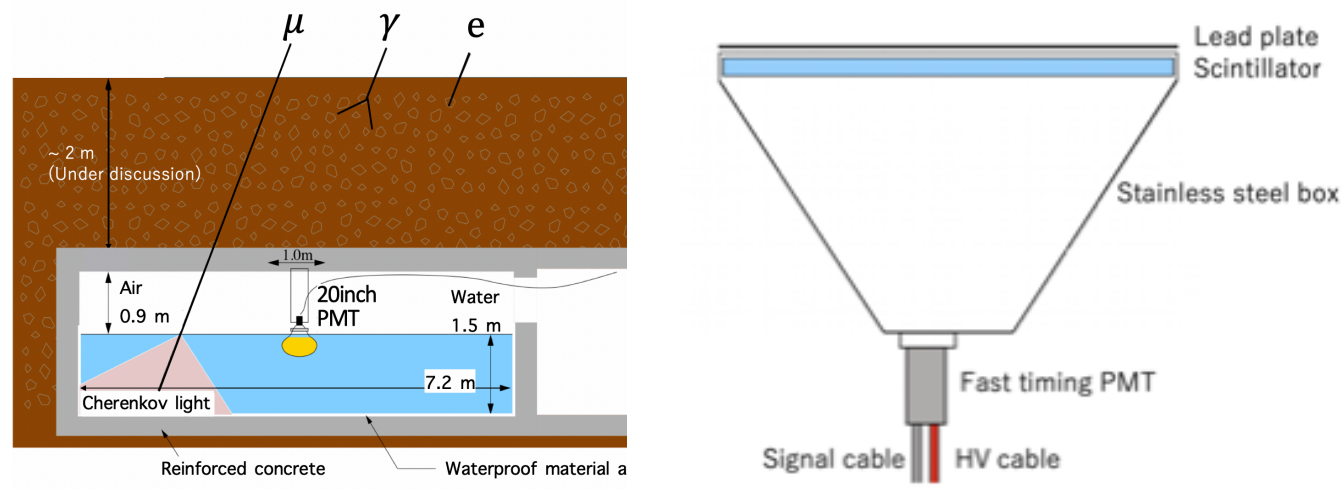

Figure 2: Left figure shows muon detector construction. Muon detector reinforced concrete structure has $2 \mathrm{~m}$ soil layer on the ceiling. The muon detector is filled with water of $1.5 \mathrm{~m}$ depth, and a 20" PMT monitors Cherekov lights produced by penetrating muon. The figure at the right figure shows air shower detector instruments.

\section{Performance of the half ALPACA}

The angular resolution is one of the barometers showing the performance air shower array. Figure3(right) gives an angular resolution of the half ALPACA for gamma-rays. Especially, at 100 $\mathrm{TeV}$ region, that value is $0.25^{\circ}$ as 50 percent containment. Above all, the angular resolution of ALPACA is similar to TibetAS $\gamma\left(0.22^{\circ} @ 100 \mathrm{TeV}\right)[9]$.

The number of muon detected allows us to estimate primary seeds. Figure 4 shows $\left(\Sigma \rho, \Sigma N_{\mu}\right)$ plot of gamma-ray and cosmic ray events. Here, $\Sigma N_{\mu}$ is defined as the total number of muon detected. The tendency that cosmic ray events are much $\Sigma N_{\mu}$ at the same $\Sigma \rho$ than gamma-ray events. We can then set the cut line(green line) and use gamma-ray events under the cutline to eliminate the enormous background cosmic ray events. Adopting that process, the rejection power of background events attains $99.9 \%$.

Sensitivity curves make us to understand that how long does it take to observe the Galactic center. Figure 5 shows the sensitivity curve of the half ALPACA. Note that the applied definition for sensitivity requires a detection significance of $5 \sigma$ per energy bin. Additional criteria are applied to require at least ten events detected gamma-rays per energy bin in the region whose background cosmic ray events are smaller than one event.In Figure5, the green line is Galactic center gamma-ray 

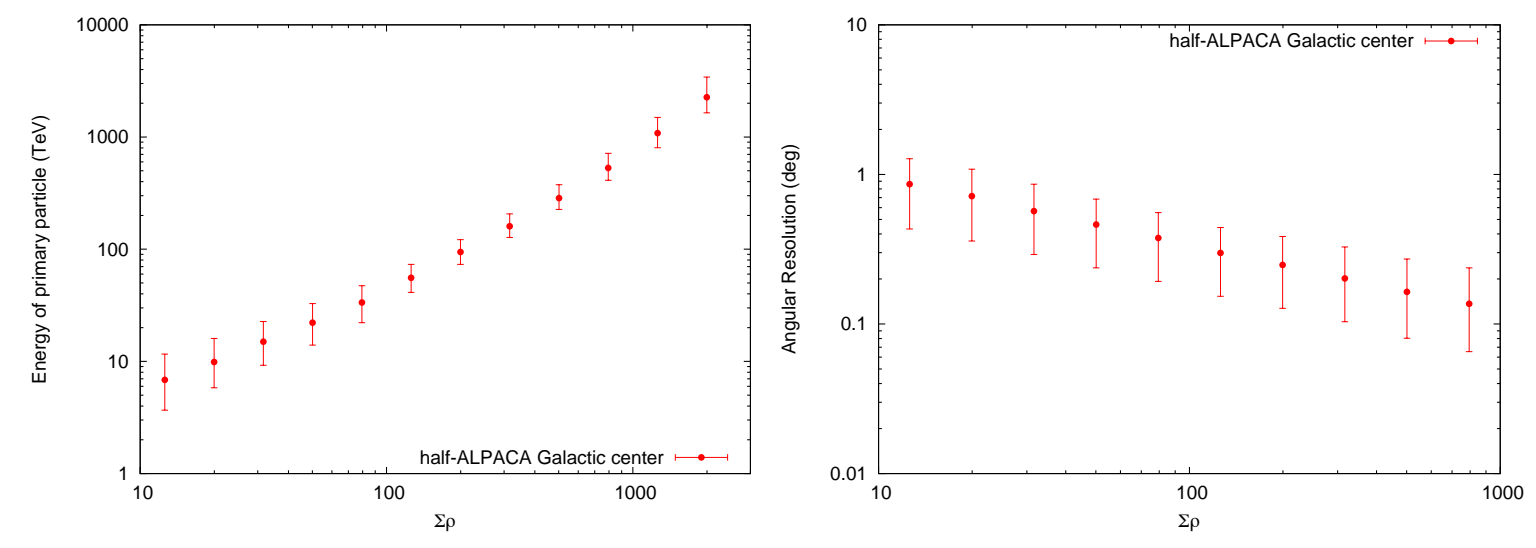

Figure 3: Left and right figure show the relationship between $\Sigma \rho$ and energy $(E)$ and the value of angular resolution versus $\Sigma \rho$. The $\Sigma \rho$ bin around 200 approximately corresponds to $100 \mathrm{TeV}$.

flux referenced H.E.S.S. observation ([3]) and black line is virtual object, the point source which has Crab gamma-ray flux and moves along the orbit of RX J1713.7-3946, gamma-ray flux. Pink line and yellow line are ALPAQUITA and ALPACA sensitivity curve. However, we estimates the ALPAQUITA and ALPACA sensitivity curves under the virtual object assumption[7].

Then, we can expect to detect the Galactic center gamma-ray flux at $100 \mathrm{TeV}$ region about 1 1.5 years observation time. 


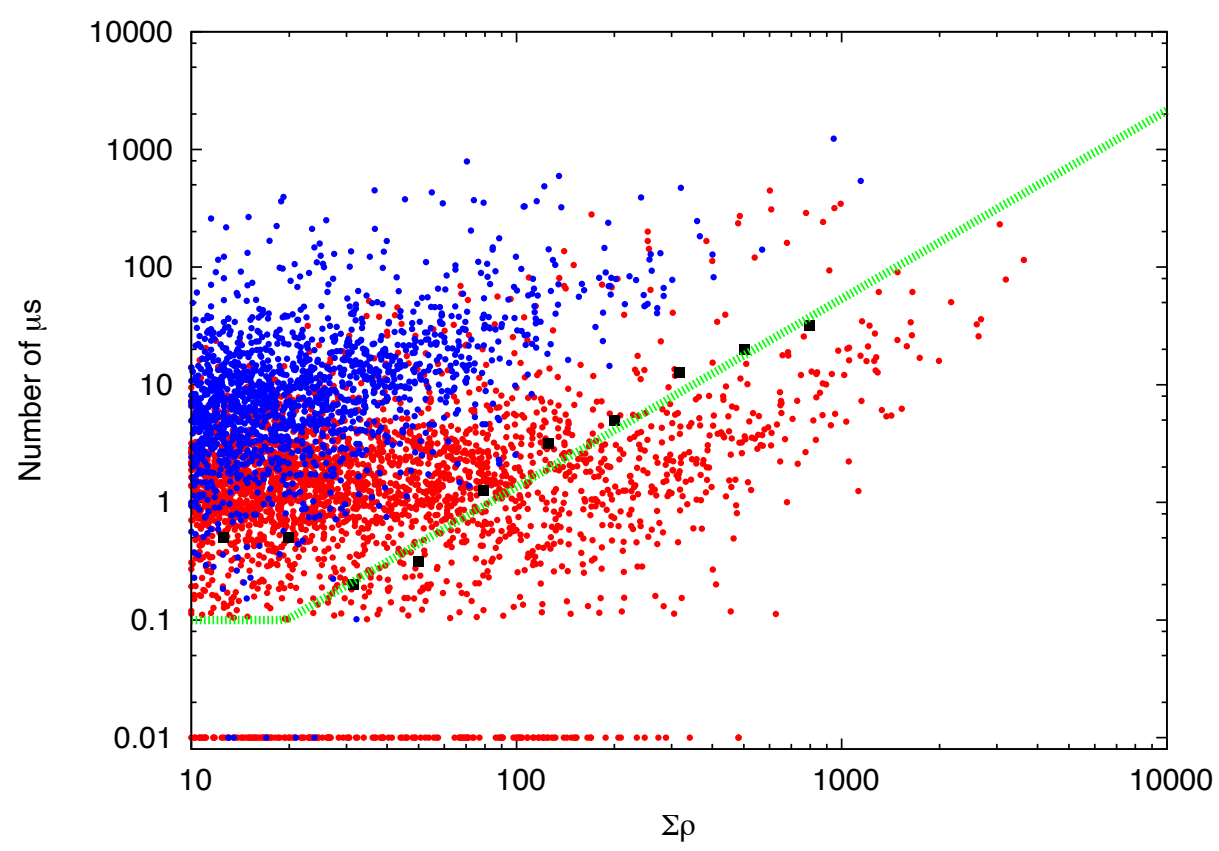

Figure 4: Red points are gamma-ray events, and blue points are cosmic ray events. In this figure, the events whose $\Sigma N_{\mu}$ is less than 0.1 accumulate $\Sigma N_{\mu}=0.01$. The green line is the cutline. In data analysis process, gamma-ray events under cutline are used.

\section{Results}

The effort of simulation in ALPACA plays an essential role in the planning and construction. In this contribution with detailed MC simulation of half ALPACA, the sensitivity curve for Galactic Center is clarified. Then, half ALPACA can be expected to observe Galactic Center gamma-ray flux in 1 1.5 years observation, but this results is estimated under the assumption of point gamma-ray source. The understanding of the half ALPACA sensitivity of the extended object is the next step.

\section{References}

[1] Daniel Gaggero, Cosmic Ray Diffusion in the Galaxy and Diffuse Gamma Emission, SpringerVerlag Berlin Heidelberg (2012)

[2] T.K.Sako et al, Exploration of a $100 \mathrm{TeV}$ gamma-ray northern sky using the Tibet air-shower array combined with an underground water-Cherenkov muon-detector array, Astroparticle physics (2009)

[3] Abramowski, A., Aharonian, F., Benkhali, F. et al. Nature, 531, 476 (2016) 


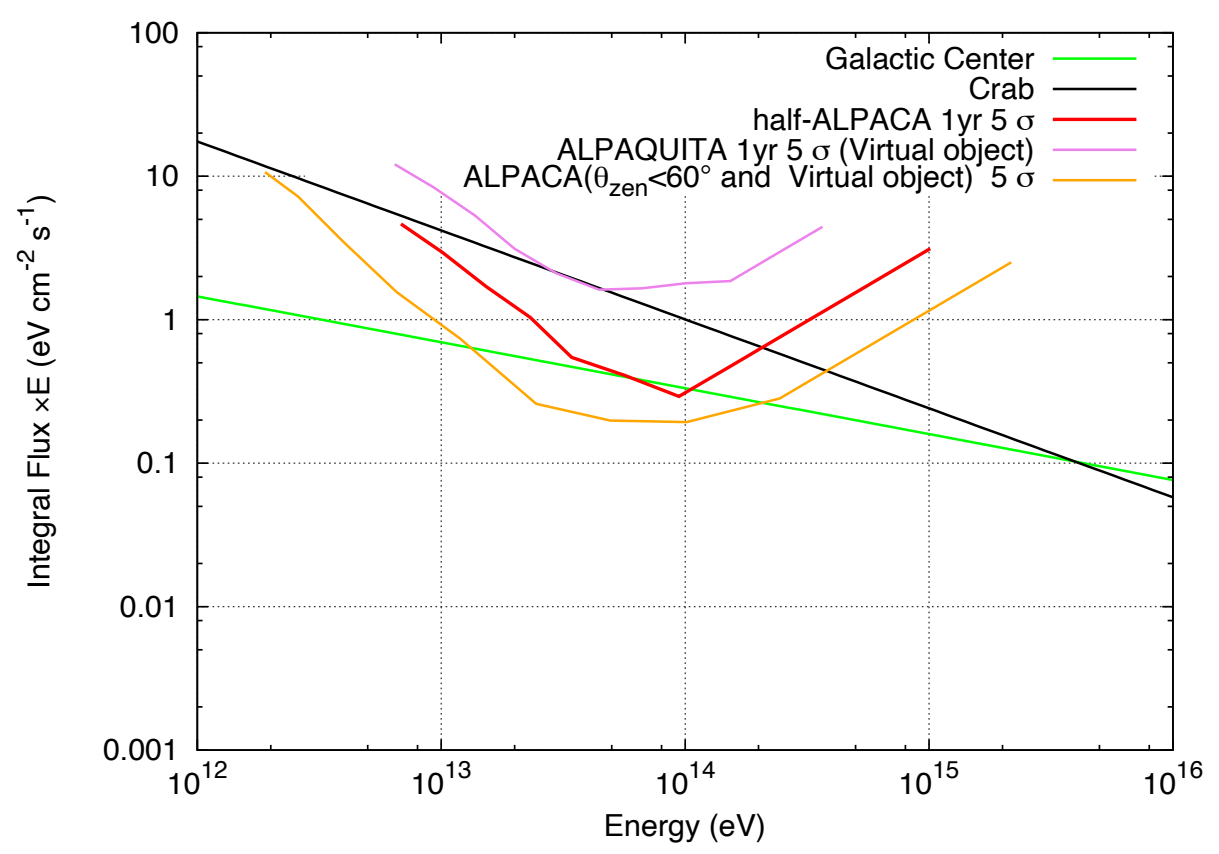

Figure 5: Half ALPACA sensitivity curve. Note that the applied definition for sensitivity requires a detection significance of $5 \sigma$ per energy bin. Additional criteria are applied to require at least ten events detected gamma-rays per energy bin in the region whose background cosmic ray events are smaller than one event. In this figure, the green line is Galactic center gamma-ray flux referenced H.E.S.S. observation([3]), and a black line is a virtual object, the point source with Crab moves along the orbit of RX J1713.7-3946, gamma-ray flux. Pink line and yellow line are ALPAQUITA and ALPACA sensitivity curves respectively. However, we estimates the ALPAQUITA and ALPACA curve under a virtual object assumption.[7]

[4] MAGIC Collaboration: V. A. Acciari et al, Astronomy and Astrophysics ,"MAGIC observations of the diffuse $\gamma$-ray emission in the vicinity of Galactic center"(2020)

[5] A. Archer et al, "TEV GAMMA-RAY OBSERVATIONS OF THE GALACTIC CENTER RIDGE BY VERITAS" arXiv:1602.08522v1 (2016)

[6] M.Shibata et al, ApJ, 716,1076 (2016)

[7] S.Kato, A simulation study on the performance of the ALPAQUITA experiment" (Oral), ICRC 2021 , this proceedings

[8] T.Sako, Current status of ALPACA for exploring sub-PeV gamma-ray sky in Bolivia, ICRC 2021, this proceedings

[9] K.Kawata, "First Detection of sub-PeV Diffuse Gamma Rays from the Galactic Disk: Evidence for Ubiquitous Galactic Cosmic Rays beyond PeV Energies",(2021) 


\section{Acknowledgements}

The ALPACA project is supported by the Japan Society for the Promotion of Science (JSPS) through Grants-in-Aid for Scientific Research (A) 19H00678, Scientific Research (B) 19H01922, and Scientific Research (S) 20H05640, the LeoAtrox supercomputer located at the facilities of the Centro de Análisis de Datos (CADS), CGSAIT, Universidad de Guadalajara, México, and by the joint research program of the Institute for Cosmic Ray Research (ICRR), The University of Tokyo. K. Kawata is supported by the Toray Science Foundation. E. de la Fuente thanks Coordinación General Académica y de Innovación (CGAI-UDG), cuerpo académico PRODEPUDG-CA-499, Carlos Iván Moreno, Cynthia Ruano, Rosario Cedano, and Diana Naylleli, for financial and administrative support during sabbatical year stay at the ICRR on 2021. I. ToledanoJuarez acknowledges support from CONACyT, México; grant 754851. 


\section{Full Authors List: ALPACA Collaboration}

F. Aceves de la Cruz ${ }^{1}$, S. Asano ${ }^{2}$, C. A. H. Condori ${ }^{3}$, E. de la Fuente ${ }^{1,4}$, J. L. Garcia-Luna ${ }^{1}$, A. Gomi ${ }^{5}$, K. Hibino ${ }^{6}$, N. Hotta ${ }^{7}$, A. Jimenez-Meza ${ }^{8}$, Y. Katayose ${ }^{9}$, C. Kato ${ }^{2}$, S. Kato ${ }^{10}$, T. Kawashima ${ }^{10}$, K. Kawata ${ }^{10}$, T. Koi ${ }^{11}$, H. Kojima ${ }^{12}$, D. Kurashige ${ }^{5}$, J. Lozoya ${ }^{13}$, R. Mayta ${ }^{14,15}$, P. Miranda ${ }^{3}$, K. Munakata ${ }^{2}$, K. Nagaya ${ }^{5}$, Y. Nakamura ${ }^{10}$, Y. Nakazawa ${ }^{16}$, C. Nina ${ }^{3}$, M. Nishizawa ${ }^{17}$, S. Ogio ${ }^{14,15}$, M. Ohnishi ${ }^{10}$, S. Okukawa ${ }^{5}$, F. Orozco ${ }^{4}$, A. Oshima ${ }^{11}$, M. Raljevich ${ }^{3}$, H. Rivera ${ }^{3}$, T. Saito ${ }^{18}$, Y. Sakakibara ${ }^{5}$, T. Sako ${ }^{10}$, T. K. Sako ${ }^{10}$, S. Shibata ${ }^{12}$, A. Shiomi ${ }^{16}$, M. Subieta ${ }^{3}$, N. Tajima ${ }^{19}$, W. Takano ${ }^{6}$, M. Takita ${ }^{10}$, Y. Tameda ${ }^{20}$, K. Tanaka ${ }^{21}$, R. Ticona ${ }^{3}$, I. Toledano-Juarez ${ }^{22,23}, \mathrm{H}$. Tsuchiya ${ }^{24}$, Y. Tsunesada ${ }^{14,15}, \mathrm{~S}$. Udo ${ }^{6}, \mathrm{~K}$. Yamazaki ${ }^{11}$ and Y. Yokoe ${ }^{10}$

${ }^{1}$ Departamento de Física, CUCEI, Universidad de Guadalajara, Guadalajara, Jalisco, Mexico. ${ }^{2}$ Department of Physics, Shinshu University, Matsumoto, Nagano 390-8621, Japan. ${ }^{3}$ Instituto de Investigaciones Físicas, Universidad Mayor de San Andrés, La Paz 8635, Bolivia. ${ }^{4}$ Doctorado en Tecnologías de la Información, CUCEA, Universidad de Guadalajara, Zapopan, Jalisco, Mexico. ${ }^{5}$ Graduate School of Engineering Science, Yokohama National University, Yokohama, Kanagawa 240-8501, Japan. ${ }^{6}$ Faculty of Engineering, Kanagawa University, Yokohama, Kanagawa 221-8686, Japan. ${ }^{7}$ Utsunomiya University, Utsunomiya, Tochigi 3218505, Japan. ${ }^{8}$ Departamento de Tecnologíias de la Información, CUCEA, Universidad de Guadalajara, Zapopan, Jalisco, Mexico. ${ }^{9}$ Faculty of Engineering, Yokohama National University, Yokohama, Kanagawa 240-8501, Japan. ${ }^{10}$ Institute for Cosmic Ray Research, University of Tokyo, Kashiwa, Chiba 277-8582, Japan. ${ }^{11}$ College of Engineering, Chubu University, Kasugai, Aichi 487-8501, Japan. ${ }^{12}$ Chubu Innovative Astronomical Observatory, Chubu University, Kasugai, Aichi 487-8501, Japan. ${ }^{13}$ Departamento de Ciencias de la Información y Desarrollo Tecnológico, Cutonala, Universidad de Guadalajara, Tonalá, Jalisco, Mexico. ${ }^{14}$ Graduate School of Science, Osaka City University, Osaka, Osaka 558-8585, Japan. ${ }^{15} \mathrm{Nambu}$ Yoichiro Institute for Theoretical and Experimental Physics, Osaka City University, Osaka, Osaka 558-8585, Japan. ${ }^{16}$ College of Industrial Technology, Nihon University, Narashino, Chiba 275-8576, Japan. ${ }^{17}$ National Institute of Informatics, Chiyoda, Tokyo 101-8430, Japan. ${ }^{18}$ Tokyo Metropolitan College of Industrial Technology, Arakawa, Tokyo 116-8523, Japan. ${ }^{19}$ RIKEN, Wako, Saitama 351-0198, Japan. ${ }^{20}$ Faculty of Engineering, Osaka ElectroCommunication University, Neyagawa, Osaka 572-8530, Japan. ${ }^{21}$ Graduate School of Information Sciences, Hiroshima City University, Hiroshima, Hiroshima 731-3194, Japan. ${ }^{22}$ Doctorado en Ciencias Físicas, CUCEI, Universidad de Guadalajara, Guadalajara, Jalisco, Mexico. ${ }^{23}$ Maestría en Ciencia de Datos, Departamento de Métodos Cuantitativos, CUCEA, Universidad de Guadalajara, Zapopan, Jalisco, Mexico. ${ }^{24}$ Japan Atomic Energy Agency, Tokai-mura, Ibaraki 319-1195, Japan. 